\title{
Surgical wound healing using hemostatic gauze scaffold loaded with nanoparticles containing sustained-release granulocyte colony-stimulating factor
}

\author{
This article was published in the following Dove Press journal: \\ International Journal of Nanomedicine \\ I December 201। \\ Number of times this article has been viewed
}

\author{
Weien Yuan ${ }^{1,2}$ \\ Zhenguo Liu' \\ 'Department of Neurology, Xinhua \\ Hospital Affiliated to Shanghai Jiao \\ Tong University School of Medicine, \\ ${ }^{2}$ School of Pharmacy, Shanghai Jiao \\ Tong University, Shanghai, People's \\ Republic of China
}

Correspondence: Zhenguo Liu Department of Neurology, Xinhua Hospital Affiliated to Shanghai Jiao Tong University School of Medicine 200092, I 665 Kongjiang Road, Shanghai, People's Republic of China

Tel/fax +86 2l 65790000

Email zhenguoliu2004@yahoo.com.cn

Weien Yuan

School of Pharmacy, Shanghai Jiao Tong University 200240, 800 Dongchuan Road, Shanghai, People's Republic of China

$\mathrm{Tel} / \mathrm{fax}+862$ I 34205072

Email yuanweien@I26.com
Background: The therapeutic strategies for malignant melanoma are still cancer chemotherapy, radiotherapy, and tumor resection. However, these therapeutic strategies often lead to a reduced neutrophilic granulocyte count or loss of more blood after surgical tumor resection. In this study, we developed a formulation of hemostatic gauze impregnated with sustained-release granulocyte colony-stimulating factor (G-CSF) with increasing of the neutrophilic granulocyte count in the blood following chemotherapy and decreasing blood loss after surgical tumor resection.

Methods: We designed a formulation with both hemostatic properties and increased neutrophil content to be used in cancer chemotherapy, radiotherapy, and tumor resection, comprising a hemostatic gauze as a scaffold and (G-CSF)-loaded dextran nanoparticles coated with polylacticco-glycolic acid (PLGA) solution fabricated by direct spray-painting onto the scaffold and then vacuum-dried at room temperature. The performance of this system was evaluated in vitro and in vivo.

Results: Nearly zero-order release of G-CSF was recorded for 12-14 days, and the cumulative release of G-CSF retained over $90 \%$ of its bioactivity in a NFS-60 cell line proliferation assay when the scaffold was incubated in phosphate-buffered saline $(\mathrm{pH} 7.4)$ at $37^{\circ} \mathrm{C}$. The in vivo hemostatic efficacy of this formulation was greater than that of native G-CSF, the scaffold directly spray-painted with G-CSF solution or PLGA organic solution as a coating, or when a blank scaffold was covered with the coating.

Conclusion: Our results suggest that this formulation has both hemostatic properties and increased neutrophil activity.

Keywords: hemostatic gauze scaffold, granulocyte colony-stimulating factor, bioactivity, sustained-release, stability

\section{Introduction}

Granulocyte colony-stimulating factor $(\mathrm{G}-\mathrm{CSF})^{1}$ is a member of a family of secreted glycoproteins which act on both neutrophils and granulocyte precursors in the bone marrow to promote their differentiation, proliferation, and maturation, to mobilize neutrophils from the bone marrow pool into the peripheral blood circulation, and to control neutrophil dynamics in the blood. ${ }^{2}$ G-CSF has been investigated as a neuroprotective agent in neurological disorders ${ }^{3-7}$ and has been used clinically to increase neutrophil levels in bone marrow transplantation, ${ }^{8}$ to aid recovery of the neutrophil count in treatment of ritodrine-induced neutropenia, ${ }^{9}$ as an adjunct to chemotherapy or radiotherapy in the treatment of cancer, ${ }^{10-13}$ and in various conditions, 
including neutropenia associated with aplastic anemia or myelodysplastic syndrome.

However, cancer chemotherapy and radiotherapy often lead to a reduced neutrophilic granulocyte count, and G-CSF is used first-line for increasing neutrophilic granulocytes in the blood. ${ }^{13}$ Clinical application of G-CSF involves frequent injections, and compliance is poor. In order to enhance compliance, the development of noninvasive dosage routes, including nasal, ${ }^{14-16}$ pulmonary, ${ }^{17-19}$ and oral ${ }^{20-22}$ formulations is expected, and investigations have been performed using various formulations, but the bioavailability of these has been very low, so they have met with little success. ${ }^{23,24}$ A long-acting injectable formulation enabling decreased dosing frequency, PEGylated G-CSF, fusion G-CSF protein, and sustained-release formulations have also been studied. ${ }^{25-27}$ However, PEGylation and fusion protein often causes G-CSF bioactivity to decrease, and sustained-release formulations suffer from loss of bioactivity and increased immunogenicity due to the methods used in their preparation, eg, a shortage of protective protein, oil-water or water-air interfaces of water-in-oil (w/o), ${ }^{28}$ water-in-oil-in-water (w/o/w), or waterin-oil-in-oil (w/o/o) emulsion methods. ${ }^{29-31}$ We developed several novel methods for preserving bioactivity of the protein by avoiding creation of oil-water or water-air interfaces, such as a stable aqueous-aqueous phase ("emulsion") and a lowtemperature aqueous-aqueous phase separation method for protein-loaded dextran nanoparticles or microparticles. ${ }^{32,33}$ These nanoparticles or microparticles may be used further to prepare sustained-release formulations. ${ }^{34-36}$ For example, the particles may be used to coat stents, scaffolds, and medical devices. Because hemostatic gauze is often used following tumor resection or in other diseases to stop bleeding, we wanted to develop a formulation of hemostatic gauze impregnated with sustained-release G-CSF. We hoped that the formulation would increase the neutrophilic granulocyte count in the blood following chemotherapy and decrease blood loss after surgical tumor resection.

The objective of this study was to develop a surgical hemostatic gauze scaffold with high encapsulating efficiency, preserved protein stability, and ability to release G-CSF in a sustained manner, by loading a hemostatic gauze scaffold with G-GSF-dextran nanoparticles and coating the scaffold with a polylactide-co-glycolic acid (PLGA) suspension. The formulation was characterized by scanning electron microscopy, size-exclusion chromatography-high-pressure liquid chromatography (HPLC), in vitro release characteristics, a NSF-60 cell proliferation assay, and in vivo efficacy.

\section{Materials and methods Materials}

PLGA (50:50, molecular weight $47 \mathrm{kDa}$; 50:50, molecular weight $20 \mathrm{kDa}$ ) was obtained from Lakeshore BioMaterials Inc (Kent, OH). Hemostatic gauze (oxidized regenerated cellulose) was purchased from Ethicon SARL (Neuchatel, Switzerland). Ethyl acetate (analytical reagent), dichloromethane (analytical reagent), dextran (molecular weight 64,000-76,000, biochemical reagent) and polyethylene glycol (PEG, molecular weight 6000 , biochemical reagent) were purchased from Sigma-Aldrich (St Louis, MO). G-CSF was supplied by Jinsai Yaoye Co Ltd (Changchun, China). A human G-CSF enzyme-linked immunosorbent assay kit was purchased from R\&D Systems Inc (Minneapolis, MN). A bipotential murine hemopoietic cell line (NFS-60) was purchased from the American Type Culture Collection (Manassas, VA).

\section{Animals}

Adult male Sprague Dawley rats weighing approximately $200 \mathrm{~g}$ were used. The animals were maintained under standardized rodent conditions at a room temperature of $22^{\circ} \mathrm{C} \pm 1{ }^{\circ} \mathrm{C}$. The animal experiments complied with the principles of laboratory animal care and were approved by the Institutional Animal Care and Utilization Committee of Shanghai Jiao Tong University.

\section{G-CSF-loaded dextran nanoparticles}

A $3 \mathrm{~mL}$ mixture of dextran $(1 \%, \mathrm{w} / \mathrm{w})$, G-CSF $(0.25 \%$, $\mathrm{w} / \mathrm{w})$, and PEG $(5 \%, \mathrm{w} / \mathrm{w})$ in a ratio of $1: 4: 20$ was prepared, ${ }^{32}$ and then frozen in a refrigerator at $-80^{\circ} \mathrm{C}$ overnight. Samples were lyophilized using a Christ Alpha 1-2 laboratory freeze-dryer at a pressure of $5.25 \times 10^{-3} \mathrm{~Pa}$ for 24 hours. The lyophilized powders were washed with $5 \mathrm{~mL}$ of dichloromethane, then centrifuged at 12,000 rpm for 5 minutes (Eppendorf 5415D, Hamburg, Germany) to remove the supernatant liquid, ie, the PEG continuous phase (because G-CSF-loaded dextran nanoparticles are insoluble in dichloromethane and would precipitate in the bottom of the centrifuge tube). The washing-centrifugation procedure was repeated three times, and the G-CSF dextran nanoparticles collected were evaporated at $1.33 \mathrm{~Pa}$ and room temperature for 24 hours using a vacuum dryer (Fuma DZF-3, Shanghai Fuma Co Ltd, Shanghai, China) to remove the dichloromethane residue. The nanoparticles obtained contained less than $0.5 \%(\mathrm{w} / \mathrm{w})$ PEG after the washing process. 


\section{Hemostatic gauze scaffold}

with G-GSF-loaded dextran nanoparticles

The hemostatic gauze scaffold containing G-GSF-loaded dextran nanoparticles was prepared by spraying and painting a $2.5 \mathrm{~cm} \times 1 \mathrm{~cm}$ piece of hemostatic gauze scaffold with a suspension containing G-CSF-loaded dextran nanoparticles and PLGA ethyl acetate solution or G-CSF in aqueous solution and PLGA ethyl acetate solution at a rate of $0.25 \mathrm{~mL} /$ minute. The suspension was prepared by vortex mixing 5\% PLGA ethyl acetate solution (containing $20 \mathrm{mg}$ PLGA) and $5 \mathrm{mg}$ G-CSF-loaded dextran nanoparticles for 5 minutes. After initial drying, the hemostatic gauze scaffold with G-CSF-loaded dextran nanoparticles was put in a refrigerator at $20^{\circ} \mathrm{C}$ for at least 10 hours, followed by further evaporation at room temperature in a vacuum below 0.1 mbar for 48 hours to remove any dichloromethane residue. The hemostatic gauze scaffold containing G-CSF-loaded dextran nanoparticles was then sprayed and painted with additional G-CSF dextran nanoparticle-free PLGA (14 mg), with the outer layer free of dextran nanoparticles to improve the release kinetics of G-CSF.

After the hemostatic gauze scaffold containing G-CSFloaded dextran nanoparticles had been refrigerated at $-20^{\circ} \mathrm{C}$ and freeze-dried for 10 hours, the pure hemostatic gauze scaffold was attached and adhered to the surface of the hemostatic gauze scaffold containing G-CSF-loaded dextran nanoparticles and further evaporated in a vacuum below 0.1 mbar for 48 hours to remove any dichloromethane residue at room temperature.

\section{Scanning electron microscopy}

Scanning electron microscopy (SEM) images of the hemostatic gauze, hemostatic gauze scaffold containing G-CSF-loaded dextran nanoparticles, and the G-CSF dextran nanoparticles alone were obtained using a FEI Sirion 200 SEM (Hillsboro, OR). Prior to image scanning, the samples were coated with gold vapor under an argon atmosphere and at 5-10 keV.

\section{Size distribution of G-CSF dextran nanoparticles}

The size distribution and average particle size of the G-CSF dextran nanoparticles were detected using a particle size and shape analyzer (Ankersmid CIS-100, Edegem, Belgium). Ten milligrams of dry particles were resuspended in a quartz cell containing isopropyl alcohol with a stirring magnet bar.

\section{G-CSF encapsulation efficiency}

The hemostatic gauze scaffold containing G-CSF-loaded dextran nanoparticles was washed with $10 \mathrm{~mL}$ dichloromethane, and then centrifuged at $12,000 \mathrm{rpm}$ for 5 minutes to remove the PLGA and recover the G-CSF-loaded dextran nanoparticles, which were then redissolved in phosphatebuffered saline ( $\mathrm{pH}$ 7.4) and diluted using a G-CSF enzymelinked immunosorbent assay kit. The G-CSF encapsulation efficiency of the hemostatic gauze scaffold with G-CSFloaded dextran nanoparticles was determined using the following equation:

$$
\text { Encapsulation efficiency }(\%)=\mathrm{P} / \mathrm{P}_{\mathrm{t}} \times 100
$$

where $\mathrm{P}$ is the actual total weight of G-CSF encapsulated into the coating on the scaffold and $\mathrm{P}_{\mathrm{t}}$ is the theoretical amount of G-CSF (obtained from feeding) encapsulated into the coating. The standard deviation for drug loading was calculated based on the coating experiments which were repeated five times.

\section{Size exclusion chromatography}

Size exclusion chromatography was carried out using a HPLC system equipped with a TSK G2000SWXL size exclusion column (Shimadzu, Tokyo, Japan). Elution was performed using a peristaltic pump with $50 \mathrm{mM}$ phosphatebuffered saline $(\mathrm{pH} 7.3)$ and a flow rate of $1.0 \mathrm{~mL} /$ minute at room temperature. Absorbance of each sample was measured at a wavelength of $214 \mathrm{~nm}$. The dissolved G-CSF sample was filtered using a $0.22 \mu \mathrm{m}$ film and then injected into the HPLC system. The flow rate of the mobile phase was $1.0 \mathrm{~mL} /$ minute. The amount of monomer or aggregate G-CSF was calculated using the Chromato-Solution-Light Shimadzu software.

\section{In vitro release profile}

The $2.5 \mathrm{~cm} \times 1 \mathrm{~cm}$ hemostatic gauze scaffolds containing G-CSF-loaded dextran nanoparticles $(18 \mathrm{mg})$ were individually incubated in vials containing $2 \mathrm{~mL}$ of phosphate-buffered saline $(100 \mathrm{mM}, \mathrm{pH} 7.4)$ at $37^{\circ} \mathrm{C}$ under constant shaking. For G-CSF release, the release medium was replaced by fresh buffer on a scheduled date and the G-CSF contents were assayed using a G-CSF enzymelinked immunosorbent assay kit. For each sample, the G-CSF release experiment was repeated five times and release profiles were calculated based on the average of three experiments. 


\section{In vitro release bioactivity}

A NFS-60 cell line were cultured in RPMI 1640 (Gibco ${ }^{\circledR}$, Invitrogen, Carlsbad, CA) with $10 \%$ fetal calf serum and $5 \mathrm{ng} / \mathrm{mL}$ G-CSF. Prior to the assays, exponentially growing cells were washed free of G-CSF and resuspended in RPMI 1640 with 5\% fetal calf serum. Samples containing $5 \times 10^{4}$ cells were incubated in $100 \mathrm{~mL}$ of RPMI 1640 with $10 \%$ fetal calf serum containing solution released from hemostatic gauze scaffold containing G-CSF-loaded dextran nanoparticles. After 48 hours, growth of NFS-60 cells was determined using the MTT method. ${ }^{37}$

\section{In vivo release study}

Five male Sprague Dawley rats (6 weeks old, average weight $200 \mathrm{~g}$ ) underwent subcutaneous implantation of a hemostatic gauze scaffold loaded with native G-CSF dextran nanoparticles, a G-CSF-loaded hemostatic gauze scaffold using native G-CSF solution and PLGA suspension, or a pure hemostatic gauze scaffold at $0.15 \mathrm{mg} / \mathrm{kg}$. A $0.4 \mathrm{~mL}$ blood sample was collected using a heparinized syringe from each animal at 1, 2, 3, 4, 5, 6, 7, 8, 10, 12, and 15 days after implantation. The blood samples were centrifuged at rate of $12,000 \mathrm{rpm}$ at $4^{\circ} \mathrm{C}$ for 20 minutes, and then plasma samples were collected. Plasma G-CSF levels were determined using a G-CSF enzyme-linked immunosorbent assay kit.

\section{In vivo efficacy study}

For the in vivo efficacy study, 6-week-old Sprague Dawley rats underwent subcutaneous implantation of a hemostatic gauze scaffold containing G-CSF-loaded dextran nanoparticles, a hemostatic gauze scaffold loaded with native G-CSF solution and PLGA suspension, a subcutaneous injection of native G-CSF solution at $0.15 \mathrm{mg} / \mathrm{kg}$, or physiological saline. A $0.2 \mathrm{~mL}$ blood sample was collected from each rat using a heparinized syringe at 1, 2, 3, 4, 5, 6, 7, $8,10,12$, and 15 days after administration. Neutrophil counts were determined as described previously. ${ }^{38}$ The percentage of neutrophils was determined using a complete blood count (Beckman Coulter LH, Fullerton, CA), and the increasing ratio of neutrophils per $\mu \mathrm{L}$ of blood was measured using the neutrophil percentage of G-CSF samples divided by neutrophil percentage of physiological saline samples.

To observe the hemostatic efficacy of the hemostatic gauze scaffold containing G-CSF-loaded dextran nanoparticles, we implanted three pieces of hemostatic gauze scaffold, ie, one piece of pure hemostatic gauze scaffold, one piece containing G-CSF-loaded dextran nanoparticles, and a piece of pure hemostatic gauze scaffold adhered to the surface of a hemostatic gauze scaffold containing G-CSF-loaded dextran nanoparticles, and then measured the time to hemostasis.

\section{Statistical analysis}

The data were expressed as the mean \pm standard deviation, and statistically significant differences were sought using the Student's $t$-test. $P<0.05$ was considered to be statistically significant. Statistical analyses were carried out using SPSS software (SPSS Inc, Chicago, IL).

\section{Results \\ Morphology and size distribution}

The morphology of the samples was characterized using SEM and an Olympus $\mu 710$ camera. Figure 1 shows the morphology of the hemostatic gauze scaffolds containing different coatings at different release times. We found that the implants started to degrade on day 2 and the hemostatic gauze in the scaffold implants was completely broken down by day $7 .{ }^{39}$ Figure $2 \mathrm{~F}, \mathrm{G}$, and $\mathrm{H}$ confirm this finding. Figure $2 \mathrm{~A}$ and $\mathrm{B}$ are images for G-CSF-loaded dextran nanoparticles alone and G-CSF-loaded dextran nanoparticles recovered from the scaffold, respectively. The results show that the surfaces of both G-CSF-loaded dextran nanoparticles alone and those recovered from the scaffold were smooth and nonporous, with the same size and morphology. Figure $2 \mathrm{C}$ and $\mathrm{D}$ are images for a blank hemostatic gauze scaffold and a scaffold containing G-CSF-loaded dextran nanoparticles, respectively. The hemostatic gauze scaffold had a fibrous structure in which groups of fine fibers formed larger bundles of fibers. The hemostatic gauze scaffold containing G-CSF-loaded dextran nanoparticles regardless of 5\% G-CSF-loaded dextran nanoparticles
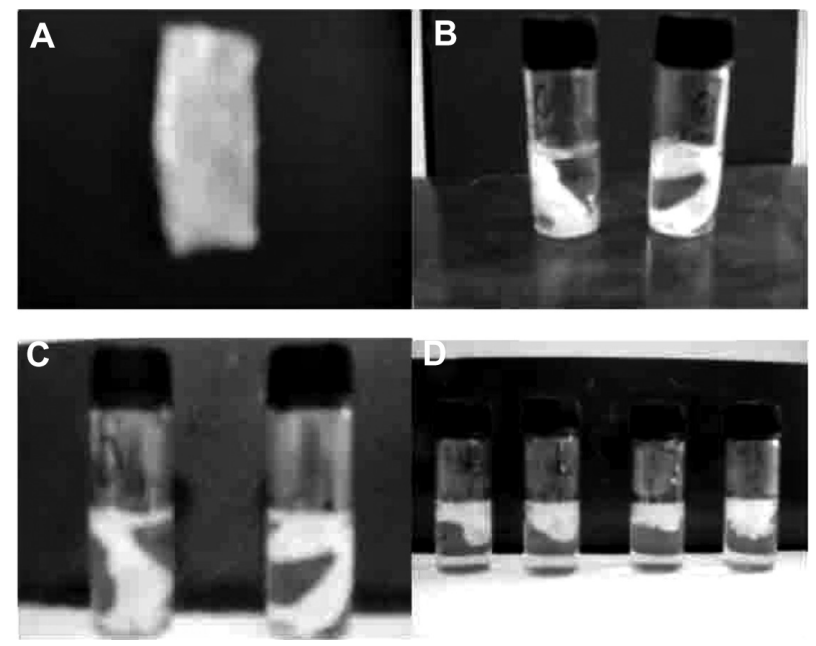

Figure I Hemostatic gauze scaffold implants. (A) Implant, (B) release on day I, (C) release on day 2 , and (D) release on day 7 . 

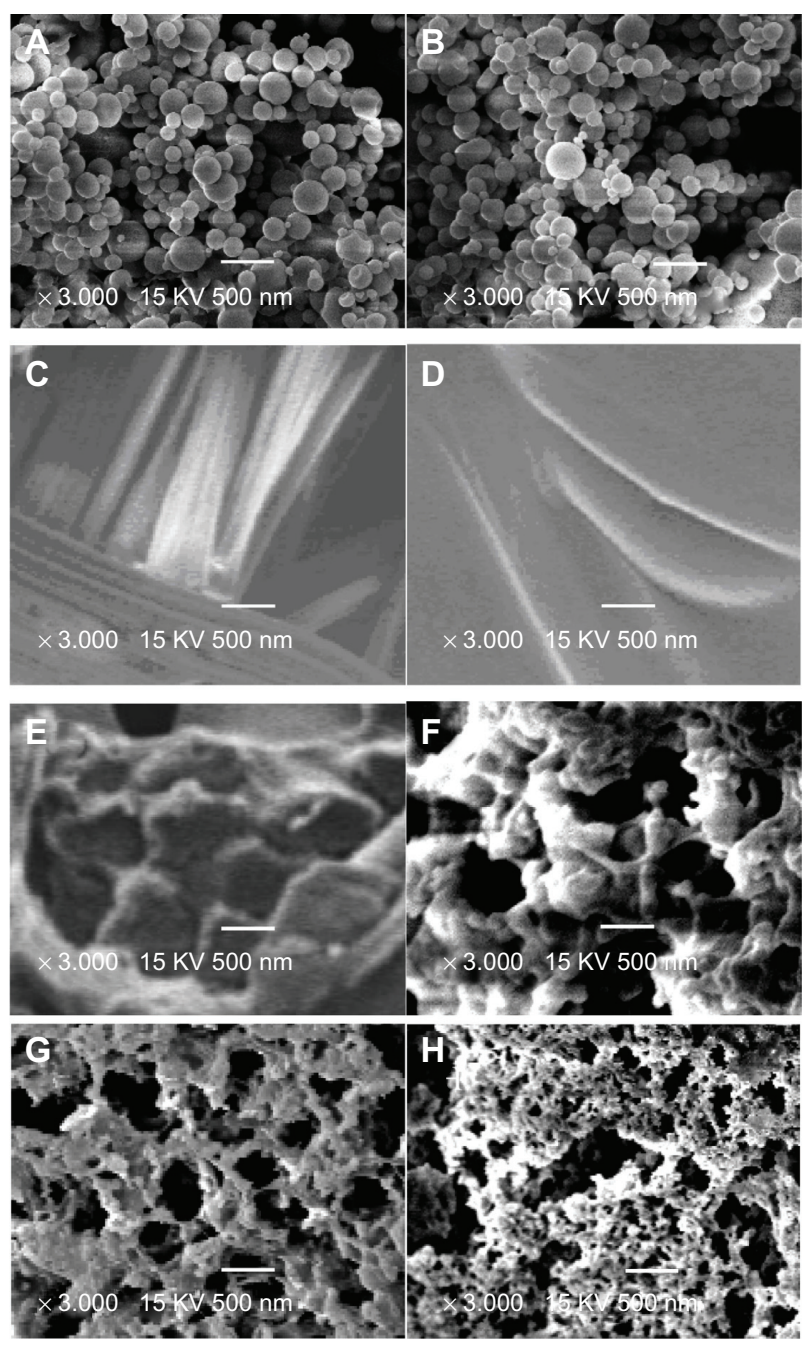

Figure 2 Scanning electron micrographs of different samples (A) G-CSF dextran nanoparticles, (B) G-CSF dextran nanoparticles from hemostatic gauze scaffold containing G-CSF-loaded dextran nanoparticles, (C) blank hemostatic gauze scaffold, (D) hemostatic gauze scaffold containing G-CSF-loaded dextran nanoparticles, and (E) cross-section from hemostatic gauze scaffold containing G-CSF-loaded dextran nanoparticles. $(\mathbf{F})$ release on day I, $(\mathbf{G})$ release on day 2, and $(\mathbf{H})$ release on day 7 . Abbreviation: G-CSF, granulocyte-colony stimulating factor.

and PLGA lead to complete coverage of the tops of the fiber bundles, and as a result, the fibrous structure became invisible (Figure 2D). A cross-section of a hemostatic gauze scaffold containing G-CSF-loaded dextran nanoparticles showed that the nanoparticles and PLGA contained gaps (Figure 2E). Figure $2 \mathrm{~F}, \mathrm{G}$, and $\mathrm{H}$ showed that the scaffold itself degraded and became more porous. The mean diameter of the G-CSF-loaded dextran nanoparticles was $100 \pm 20 \mathrm{~nm}$, with the majority of nanoparticles ranging from 60 to $100 \mathrm{~nm}$.

\section{G-CSF encapsulation efficiency from coating of hemostatic gauze implants}

G-CSF encapsulation efficiency was measured using a human G-CSF enzyme-linked immunosorbent assay kit.
The encapsulation efficiency of the first-step particles, ie, loading the proteins into the dextran nanoparticles, was above $95 \%(\mathrm{w} / \mathrm{w}),{ }^{32}$ and also for the subsequent two steps. The G-CSF loading yield was $96 \%$ for the samples projecting $1 \%$ G-CSF loads (ie, G-CSF reached $1 \%$ of the total mass of the coating material).

\section{Aggregation study in hemostatic gauze scaffolds containing G-CSF-loaded dextran nanoparticles}

Formation of protein aggregates gives a good indication of protein integrity during the microencapsulation process. ${ }^{40}$ Therefore, we assayed the samples using size-exclusion chromatography-HPLC in order to determine any change in G-CSF with the different procedures. Figure 3 shows the size-exclusion chromatography-HPLC results for G-CSF aggregation after each preparation step. Figure $3 \mathrm{~A}, \mathrm{~B}, \mathrm{C}$, and $\mathrm{D}$ show the monomer contents of the original G-CSF solution, G-CSF from the dextran nanoparticles, recovery from hemostatic gauze scaffold containing G-CSF-loaded dextran nanoparticles, and from hemostatic gauze scaffold loaded directly with G-CSF solution and PLGA suspension, respectively. The monomer content of G-CSF did not decrease during the coating process compared with the original sample, except that the monomer content of G-CSF in the hemostatic gauze scaffold loaded with G-CSF decreased by $20 \%$. These results strongly suggest that the implant preparation process used in the present study is a useful method for coating hemostatic gauze scaffold implants without aggregation.

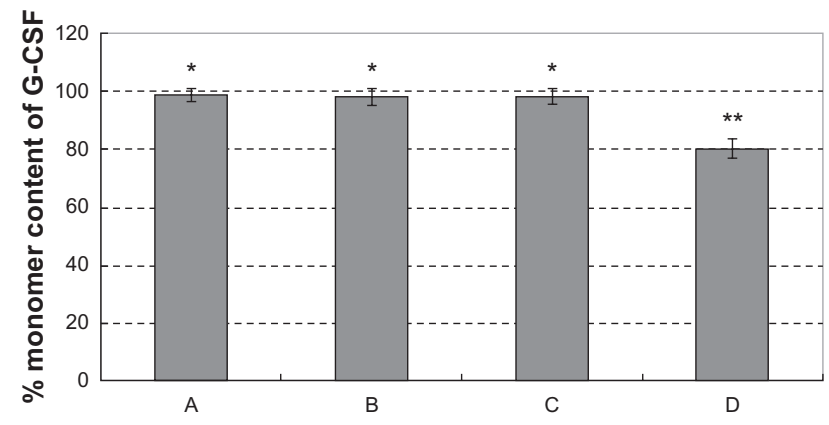

Figure 3 Monomer content of recovery G-CSF from the scaffold by size exclusion chromatography-high pressure liquid chromatography $(n=5, * P>0.05$, $* * P<0.05)$. (A) G-CSF (G-CSF:dextran = 1.4:5.0 $\pm 0.3 \mathrm{mg}$ ) solution; (B) G-CSF from G-CSFloaded dextran nanoparticles; (C) G-CSF from hemostatic gauze scaffold containing G-CSF-loaded dextran nanoparticles (PLGA 50/50 3A: first coating $=20.0 \pm 0.5 \mathrm{mg}$ PLGA, G-CSF-loaded dextran nanoparticles (G-CSF:dextran, I:4) $=5.0 \pm 0.3 \mathrm{mg}$, second coating (blank) =14.0 $\pm 0.6 \mathrm{mg}$ PLGA); (D) G-CSF from hemostatic gauze scaffold containing G-CSF-loaded dextran nanoparticles (PLGA 50/50 3A: first coating $=20.0 \pm 0.5 \mathrm{mg}$ PLGA, G-CSF solution [G-CSF:dextran = I:4] $=5.0 \pm 0.3 \mathrm{mg}$, second coating [blank] = $14.0 \pm 0.6 \mathrm{mg}$ PLGA).

Abbreviations: G-CSF, granulocyte-colony stimulating factor; PLGA, polylacticco-glycolic acid. 


\section{Bioactivity of hemostatic gauze scaffold containing G-CSF-loaded dextran nanoparticles}

In order to determine the bioactivity of the different samples, G-CSF was recovered from the formulations according to the method used for determination of G-CSF encapsulation efficiency. The bioactivity of G-CSF was assayed using the NFS-60 cell line. Figure 4 shows the bioactivity of G-CSF from the different samples, respectively. Figure $4 \mathrm{~A}, \mathrm{~B}$, and $\mathrm{C}$ shows the bioactivity of the original G-CSF solution, G-CSF from G-CSF-loaded dextran nanoparticles, and G-CSF from the hemostatic gauze scaffold containing G-CSF-loaded dextran nanoparticles (PLGA 50/50 3A), respectively. We found that the bioactivity of the samples was almost the same as for the original G-CSF solution $(P>0.05)$. Figure 4D, E, and F show the bioactivity of G-CSF from a hemostatic gauze scaffold containing G-CSF-loaded dextran nanoparticles (PLGA 50/50 1A), a hemostatic gauze scaffold loaded with G-CSF solution (PLGA 50/50 3A), and a hemostatic gauze scaffold loaded directly using G-CSF solution and PLGA suspension (PLGA 50/50 1A), respectively. The results show that the recovered bioactivity of G-CSF from a hemostatic gauze scaffold containing G-CSF-loaded dextran nanoparticles (PLGA 50/50 1A), a hemostatic gauze scaffold loaded with G-CSF solution and PLGA suspension (PLGA 50/50 3A and PLGA 50/50 1A, respectively) was decreased by $17 \%, 55 \%$, and $60 \%$, respectively $(P<0.05)$. The recovered bioactivity suggested that the G-CSF in the G-CSF-loaded dextran nanoparticles had been well protected during the preparation process. This is probably because G-CSF-loaded dextran nanoparticles might avoid oil-water and water-gas interfaces which result in loss of G-CSF bioactivity. ${ }^{31}$

\section{Release kinetics for hemostatic gauze scaffold containing G-CSF-loaded dextran nanoparticles}

Figure 5A and B summarize the G-CSF release profiles for hemostatic gauze scaffolds containing PLGA coatings of different molecular weights. Interestingly, as shown in Figure 5, the two samples showed a similar zero order G-CSF release profile regardless of molecular weight (release kinetics equations: $\mathrm{Q}_{\mathrm{A}}=12.574 \mathrm{t}+9.4172, R^{2}=0.9705$ and $\mathrm{Q}_{\mathrm{B}}=8.90003 \mathrm{t}+7.4446, R^{2}=0.962$, from day 1 to day 7 ), but the amount of cumulative release was less from the low molecular weight polymer than from the high molecular weight polymer. We reported the result of the degradation study by plotting the weight of each formulation against the release incubation time at $37^{\circ} \mathrm{C}$. When a piece of hemostatic gauze scaffold containing no coating $(2.5 \mathrm{~cm} \times 1 \mathrm{~cm}, 18 \mathrm{mg})$ was incubated in $2 \mathrm{~mL}$ of phosphate-buffered saline at $37^{\circ} \mathrm{C}$, the total mass completely disappeared within 3 days. ${ }^{39}$ As shown in Figures 1, 2F, G, and H, hemostatic gauze scaffolds coated with G-CSF-loaded dextran nanoparticles and a PLGA layer of different molecular weights or with an additional blank PLGA layer all broke down during the same time period, suggesting that the rate of release of G-CSF

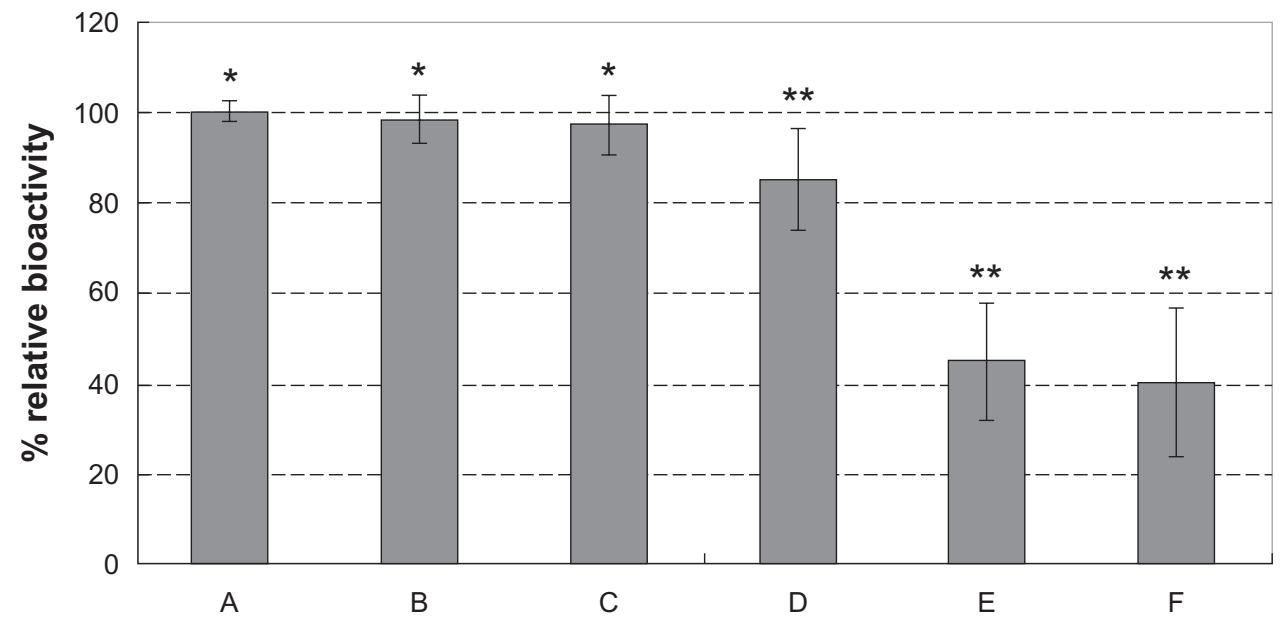

Figure 4 Recovery bioactivity from a hemostatic gauze scaffold containing G-CSF-loaded dextran nanoparticles $(\mathrm{n}=5$, $* P>0.05, * * P<0.05)$. Samples (A), (B), (C), and (E) were the same as for Figure 3A, B, C, and D, respectively $(n=5, * P>0.05$, $* * P<0.05)$. (D) Hemostatic gauze scaffold containing G-CSF from G-CSF-loaded dextran nanoparticles (PLGA 50/50 IA: first coating $=20.0 \pm 0.6 \mathrm{mg}$ PLGA, G-CSF loaded-dextran nanoparticles [G-CSF: dextran $=$ I:4] $=5.0 \pm 0.2 \mathrm{mg}$, second coating [blank] = I4.0 $\pm 0.5 \mathrm{mg}$ PLGA), (F) G-CSF from hemostatic gauze scaffold containing G-CSF-loaded dextran nanoparticles (PLGA 50/50 IA: first coating = $20.0 \pm 0.6 \mathrm{mg}$ PLGA, G-CSF solution [G-CSF:dextran = I:4] = $5.0 \pm 0.2 \mathrm{mg}$, second coating [blank] = $14.0 \pm 0.5 \mathrm{mg}$ PLGA).

Abbreviations: G-CSF, granulocyte-colony stimulating factor; PLGA, polylactic-co-glycolic acid. 


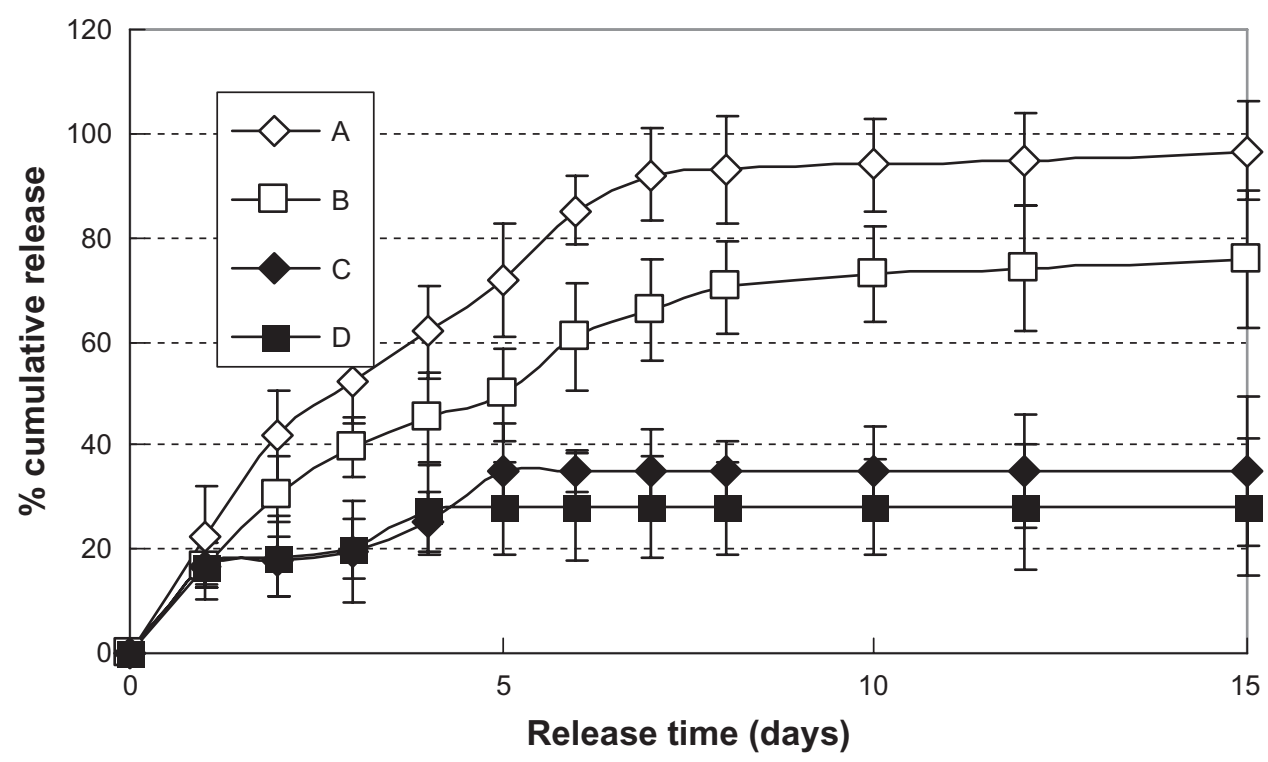

Figure 5 In vitro release profiles of hemostatic gauze scaffold containing G-CSF-loaded dextran nanoparticles $(n=5, P<0.05)$. Samples $(\mathbf{A})$, (B), (C), and (D) were the same as for Figure 3(C), (D), (E), and (F), respectively.

was due to degradation of the hemostatic gauze scaffold and independent of the PLGA coating. When G-CSF solution was loaded into hemostatic gauze scaffold using PLGA coating, the percentage cumulative release was only $30 \%-40 \%$. This was probably because the spraying and painting process produced a number of oil-water and gas-water interfaces, resulting in G-CSF aggregation and more hydrophobicity, with no release of $\mathrm{G}-\mathrm{CSF}^{31}$

\section{In vitro G-CSF release bioactivity in hemostatic gauze scaffold containing G-CSF-loaded dextran nanoparticles}

The bioactivity of in vitro release from hemostatic gauze scaffolds containing G-CSF-loaded dextran nanoparticles was investigated using the NFS-60 cell line. Figure 6A and $\mathrm{B}$ show that the bioactivity of release of G-CSF from hemostatic gauze scaffolds containing G-CSF-loaded dextran nanoparticles (PLGA 50/50 3A and PLGA 50/50 1A) showed high efficiency. The release bioactivity percent of G-CSF on the first day was $20 \%$ of the cumulative release bioactivity of G-CSF, and the percent of cumulative release bioactivity was about $80 \%-96 \%$ of the total bioactivity within the scaffold coating. When the G-CSF solution was loaded into hemostatic gauze scaffolds with PLGA coating, the percent cumulative release bioactivity of G-CSF was only $30 \%-40 \%$. This was probably because the spraying and painting process produced a number of oil-water and gas-water interfaces resulting in G-CSF aggregation and more hydrophobicity. ${ }^{31}$

\section{Release of G-CSF in an in vivo rat study} We examined the release of G-CSF from the G-CSF-loaded dextran nanoparticles and from a hemostatic gauze scaffold loaded directly using G-CSF solution and PLGA suspension, because the G-CSF released is only bioactive in vivo. Figure 7 $\diamond$ and $\diamond$ show the plasma G-CSF concentration-time release profiles for G-CSF-loaded dextran nanoparticles and hemostatic gauze scaffold loaded directly with G-CSF solution and PLGA suspension, respectively. The results showed a stable plasma concentration from days 1 to 7 , and then slow release of G-CSF up to day 15 from hemostatic gauze scaffolds containing G-CSF-loaded dextran nanoparticles. However, scaffolds loaded directly with G-CSF solution and PLGA suspension did not show stable plasma concentrations, with fast release of G-CSF from day 1 to day 3, and no G-CSF release thereafter. This confirms that the hemostatic gauze scaffold containing G-CSF-loaded dextran nanoparticles was more effective than the scaffold containing the directly loaded G-CSF solution.

\section{In vivo efficacy study}

To compare the in vivo efficacy of scaffolds containing G-CSF-loaded dextran nanoparticles, scaffolds containing directly loaded G-CSF solution, pure scaffolds, and native G-CSF alone, male Sprague Dawley rats underwent subcutaneous implantation with scaffolds loaded with dextran nanoparticles, scaffolds loaded with G-CSF, pure scaffolds, or subcutaneous injection of native G-CSF at $0.15 \mathrm{mg} / \mathrm{kg}$. Changes in neutrophil count were determined 


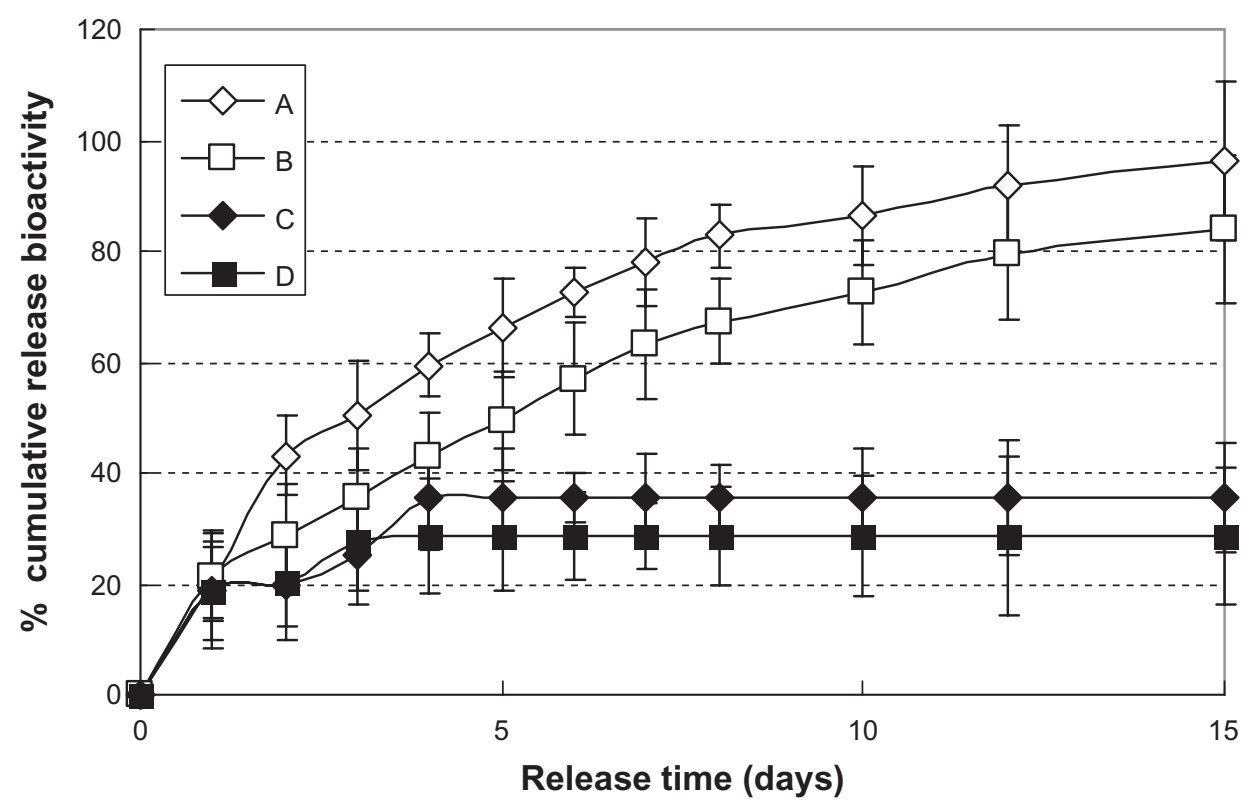

Figure 6 In vitro release relative bioactivity of hemostatic gauze scaffold containing G-CSF-loaded dextran nanoparticles $(\mathrm{n}=5, P<0.05)$. Samples $(\mathbf{A}),(\mathbf{B}),(\mathbf{C})$, and $(\mathbf{D})$ were the same as for Figure 3(C), (D), (E), and (F), respectively.

Abbreviation: G-CSF, granulocyte-colony stimulating factor.

for 15 days. The time courses in Figure $8 \boldsymbol{\Delta}, \diamond$, and $\bullet$ show that scaffolds containing G-CSF-loaded dextran nanoparticles or G-CSF solution achieved a significant increase in neutrophil count for a prolonged period of time, but the increasing ratio of neutrophil count and prolonged time from G-CSF solution-loaded the scaffold were more than G-CSF solution-loaded the scaffold (the pure scaffold did not show an increase in neutrophil count, data not shown).

We found that the time to hemostasis for the scaffold containing G-CSF-loaded dextran nanoparticles was longer than that for the same-sized pure hemostatic gauze scaffold and pure hemostatic gauze scaffold adhered to a scaffold loaded with dextran nanoparticles. However, the time to hemostasis from both a pure hemostatic gauze scaffold and a pure hemostatic gauze scaffold adhered to the surface of a scaffold loaded with dextran nanoparticles was almost the same $(P<0.05)$. This suggests that the scaffold loaded with dextran nanoparticles might work for both increasing the neutrophil count and stopping bleeding when the pure hemostatic gauze scaffold was attached and adhered to the surface of a scaffold loaded with dextran nanoparticles.

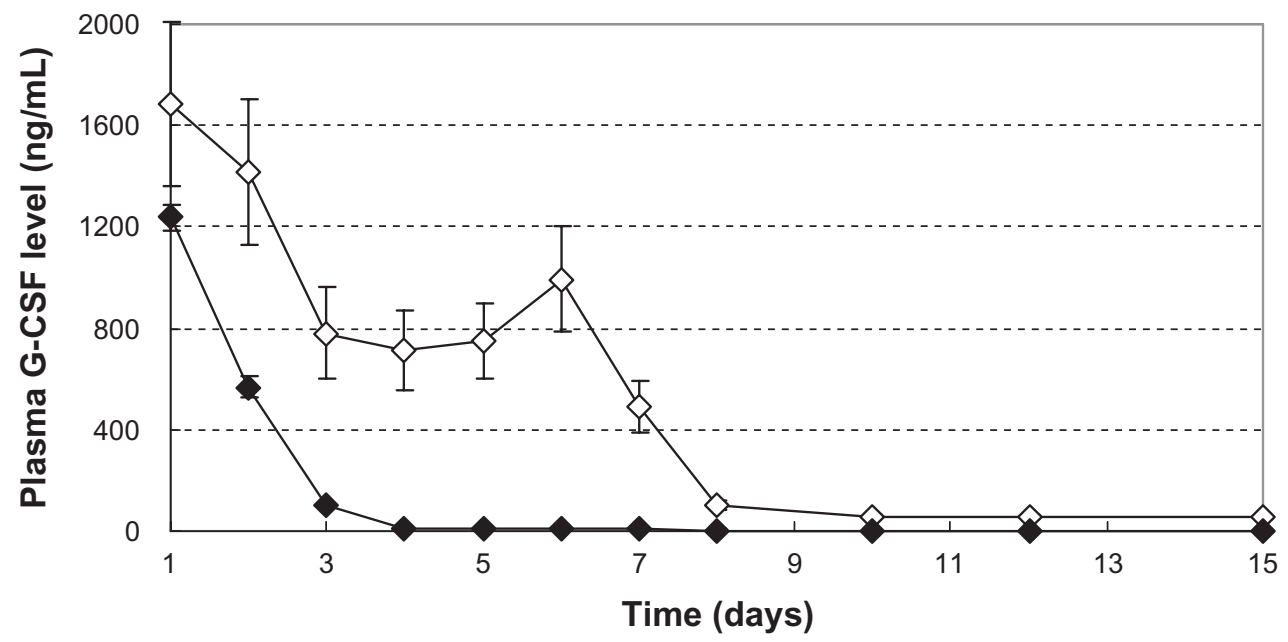

Figure 7 In vivo release plasma G-CSF level of hemostatic gauze scaffold containing G-CSF-loaded dextran nanoparticles ( $n=5, P<0.05)$. Samples $\diamond$ and $\diamond$ were the same as for Figure 3(C) and (E), respectively.

Abbreviation: G-CSF, granulocyte-colony stimulating factor. 


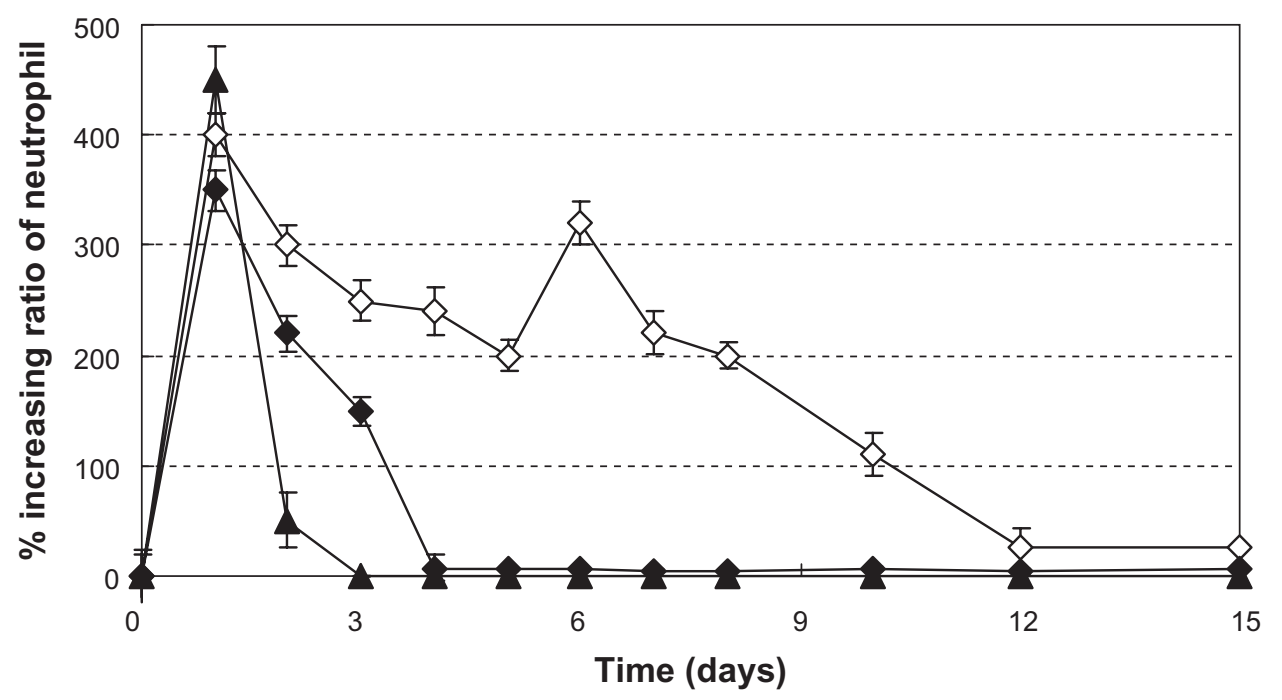

Figure 8 Increasing ratio of in vivo neutrophil levels $(n=5, P<0.05)$. $\boldsymbol{\Delta}, \diamond$, and $\diamond$ were the same as for Figure $3(\mathbf{A}),(\mathbf{C})$, and $(\mathbf{E})$, respectively.

\section{Discussion}

A zero order release profile for G-CSF was observed from the scaffolds containing G-CSF-loaded dextran nanoparticles, regardless of the molecular weight of the PLGA coating used from days 1 to 7 (Figures 5A and B). This finding suggests that the rate-limiting step for G-CSF release was not just controlled by PLGA, but by other factors, including the dextran nanoparticles and the scaffold. The pure scaffold is easily degraded, taking 1-2 weeks to break down into molecular pieces (http://store.k12webstore.com/mkgz24. html), and dextran nanoparticles also absorb water and swell..$^{43}$ These observations suggest that pores or holes in the surface of the PLGA coating can be created when a pure hemostatic gauze scaffold with PLGA is degraded and broken fragments form. ${ }^{39}$ Rapid degradation of the hemostatic gauze scaffold creates sufficient penetration of water diffusion channels for G-CSF release of all implants coated with PLGA of different molecular weights. ${ }^{39}$ At the same time, the G-CSF diffusion distance from the inner to outer coating decreases due to swelling of the dextran nanoparticles through absorption of water compared with no swelling additives. ${ }^{42}$

These resulting in that at the beginning of the release phase, G-CSF were near the coating surface of the G-CSFloaded dextran nanoparticle scaffolds, then the hemostatic gauze scaffold started to degrade and formed more and more new holes in the coating that led to water being permeated into the coating and increased diffusion of G-CSF and dextran swelling (of course, finally dextran also was dissolved and diffused, the PLGA also was degraded, but PLGA degraded slower than the pure scaffold during the whole release process). With these diffusion channels, the diffusion rate of G-CSF became significantly higher than that of high molecular weight dextran, thus the overall release rate was limited by the swelling of dextran. Figure $2 \mathrm{~F}-\mathrm{H}$ also confirmed the process. ${ }^{39}$ The linear release from the G-CSFloaded dextran nanoparticle scaffold appeared attributable to the above effects. ${ }^{44-46}$

Figures $5 \mathrm{~A}$ and $\mathrm{B}, 6 \mathrm{~A}$ and $\mathrm{B}$ also show that the degradation rate of PLGA did not affect the release rate or cell proliferation activity of the G-CSF released or the cumulative amount released from the scaffolds containing G-CSF-loaded dextran nanoparticles. The cumulative amount released from scaffolds containing G-CSF-loaded dextran nanoparticles with PLGA of higher molecular weight was more than that of PLGA of lower molecular weight. This was possibly because G-CSF was unstable in a locally acidic environment, ${ }^{41,43}$ in which the degradation rate of lower molecular weight PLGA was faster than for higher molecular weight PLGA which, in turn, formed a more acidic local environment. ${ }^{41,43}$ Figure 5C and $\mathrm{D}$ show further release kinetic profiles which were probably because G-CSF had aggregated and become more hydrophobic, resulting in no release of $\mathrm{G}-\mathrm{CSF},{ }^{43}$ suggesting that the spray-painted coatings had produced a number of oil-water and gas-water interfaces. ${ }^{31}$

In order to understand the mechanism of the scaffold, a pure hemostatic gauze scaffold was attached and adhered to the surface of a hemostatic gauze scaffold containing G-CSFloaded dextran nanoparticles for study of in vitro and in vivo release. We found that the release profile, plasma G-CSF level, and increase in neutrophil count were the same for a hemostatic gauze scaffold containing G-CSF-loaded dextran nanoparticles (data no shown), but that the time to hemostasis 
was shorter. This suggested that the pure hemostatic gauze scaffold does not affect the activity of the G-CSF-loaded dextran nanoparticle scaffold in terms of release of G-CSF, but may improve hemostasis activity.

By coating the gauze scaffold with G-CSF-loaded dextran nanoparticles and PLGA suspension, sustained-release delivery of the drug can be achieved easily. In addition, the desired release kinetics, such as a low level of initial burst release and a zero order release profile can also be easily achieved. With the right thickness, the G-CSF-loaded dextran nanoparticle-free top layer of PLGA may provide additional control of G-CSF release mechanisms. The PLGA coating on the hemostatic gauze scaffold can easily be attached and applied to the wound surface during surgery to stop post-surgical bleeding and to improve the neutrophilic granulocyte count in blood during chemotherapy as well as to staunch bleeding after tumor resection.

\section{Conclusion}

Using a clinically available hemostatic agent as a scaffold, we demonstrated that a hemostatic gauze scaffold containing G-CSF-loaded dextran nanoparticles can achieve controlled delivery of G-CSF. G-CSF were released from this formulation through a mechanism of diffusion channels independent of G-CSF-loaded different molecular weight PLGA that was created due to rapid degradation of the hemostatic gauze scaffold inside the PLGA coating and dextran microparticle swelling. Such diffusion channels could be blocked by an additional coating of G-CSF-loaded dextran nanoparticlefree PLGA to reach the desired G-CSF release rate. In addition to nearly zero order and burst-free release kinetics, this formulation approach was simple and did not require a complicated microencapsulation process, thus could easily be scaled up and potentially used to improve the neutrophilic granulocyte level in blood at the time of chemotherapy and staunch bleeding after tumor resection.

\section{Acknowledgments}

The study was supported by the Project of National Science Foundation of China Committee (81071025, 81171203), the PhD Programs Foundation of Ministry of Education of China (20090073120085), and the University Student Renovation Project of Shanghai Jiao Tong University (IPP2090). We also appreciate the generous help of the Instrumental Analysis Centre of Shanghai Jiao Tong University.

\section{Disclosure}

The authors declare no competing financial interests in this work.

\section{References}

1. Kamachi S, Motojima H, Oheda M, et al. Properties of natural granulocyte colony-stimulating factor (G-CSF). Kiso to Rinsho Clinical Report. 1991;25:311-316.

2. Shochat E, Rom-Kedar V, Segel LA. G-CSF control of neutrophils dynamics in the blood. Bull Math Biol. 2007;69:2299-2338.

3. Frank T, Schlachetzki JC, Göricke B, et al. Both systemic and local application of granulocyte-colony stimulating factor (G-CSF) is neuroprotective after retinal ganglion cell axotomy. BMC Neurosci. $2009 ; 10: 49$

4. McCollum M, Ma Z, Cohen E, et al. Post-MPTP treatment with granulocyte colony-stimulating factor improves nigrostriatal function in the mouse model of Parkinson's disease. Mol Neurobiol. 2010;41: 410-419.

5. Pitzer C, Klussmann S, Krüger C, et al. The hematopoietic factor granulocyte-colony stimulating factor improves outcome in experimental spinal cord injury. J Neurochem. 2010;113:930-942.

6. Schlager GW, Griesmaier E, Wegleiter K, et al. Systemic G-CSF treatment does not improve long-term outcomes after neonatal hypoxicischaemic brain injury. Exp Neurol. 2011;230:67-74.

7. Bartolini A, Vigliani M-C, Magrassi L, Vercelli A, Rossi F. G-CSF administration to adult mice stimulates the proliferation of microglia but does not modify the outcome of ischemic injury. Neurobiol Dis. 2011;41:640-649.

8. Lieschke GJ, Ramenghi U, O'Connor MP, Sheridan W, Szer J, Morstyn G. Studies of oral neutrophil levels in patients receiving G-CSF after autologous marrow transplantation. Br J Haematol. 1992;82: 589-595.

9. Kikkawa M, Matsubara S, Takatoku M, et al. Granulocyte-colony stimulating factor for the treatment of ritodrine-induced neutropenia. J Obstet Gynaecol Res. 2008;34:286-290.

10. Kantariian HM, Estey E, O'Brien S, et al. Granulocyte colony-stimulating factor supportive treatment following intensive chemotherapy in acute lymphocytic leukemia in first remission. Cancer. 1993;72: 2950-2955.

11. Felici A, Carlini P, Ruggeri EM, et al. Bi-weekly chemotherapy with cisplatin, epirubicin, folinic acid and 5-fluororacil continuous infusion plus G-CSF in advanced gastric cancer: A multicentric phase II study. Cancer Chemother Pharmacol. 2006;57:59-64.

12. Russell H, Shohe JM. Pediatric oncology: G-CSF counteracts chemotherapy toxicity in neuroblastoma. Nat Rev Clin Oncol. 2011; $8: 6-8$

13. Crea F, Giovannetti E, Zinzani PL, Danesi R. Pharmacologic rationale for early G-CSF prophylaxis in cancer patients and role of pharmacogenetics in treatment optimization. Crit Rev Oncol Hematol. 2009;72: 21-44.

14. Machida M, Sano K, Arakawa M, Hayashi M, Awazu S. Absorption of recombinant human granulocyte colony- stimulating factor (rhG-CSF) from rat nasal mucosa. Pharm Res. 1993;10:1372-1377.

15. Nomura H, Akamisaka S, Maruyama K. Effects of a dosing solution on the nasal absorption of non-glycosylated recombinant human granulocyte colony-stimulating factor in rats. Biol Pharm Bull. 1996;19: $1490-1493$

16. Watanabe Y, Kikuchi R, Kiriyama M, et al. Increase in total blood leukocyte count following intranasal administration of recombinant human granulocyte colony-stimulating factor (rhG-CSF) in rabbits with cyclophosphamide-induced leucopenia. Biol Pharm Bull. 1995;18: 1084-1088.

17. Machida M, Hayashi M, Awazu S. The effects of absorption enhancers on the pulmonary absorption of recombinant human granulocyte colony-stimulating factor (rhG-CSF) in rats. Biol Pharm Bull. 2000;23: 84-86.

18. Machida M, Hayashi M, Awazu S. Pulmonary absorption of recombinant human granulocyte colony-stimulating factor (rhG-CSF) after intratracheal administration to rats. Biol Pharm Bull. 1996;19: 259-262. 
19. Niven RW, Whitcomb KL, Shaner L, Ip AY, Kinstler OB. The pulmonary absorption of aerosolized intratracheally instilled rhG-CSF and monoPEGylated rhG-CSF. Pharm Res. 1995;12:1343-1349.

20. Eiamtrakarn S, Itoh Y, Kishimoto J, et al. Gastrointestinal mucoadhesive patch system (GI-MAPS) for oral administration of G-CSF, a model protein. Biomaterials. 2002;23:145-152.

21. Takaya T, Ikeda C, Imagawa N, Niwa K, Takada K. Development of a colon delivery capsule and the pharmacological activity of recombinant human granulocyte colony stimulating factor (rhG-CSF) in beagle dogs. J Pharm Pharmacol. 1995;47:474-478.

22. Jensen-Pippo KE, Whitcomb KL, DePrince RB, Ralph L, HabberfieldAD. Enteral bioavailability of human granulocyte colony-stimulating factor conjugated with poly(ethyleneglycol). Pharm Res. 1996;13: $102-107$.

23. Shen WC. Oral peptide and protein delivery: unfulfilled promises? Drug Discov Today. 2003;8:607-608.

24. Lee HJ. Protein drug oral delivery: the recent progress. Arch Pharm Res. 2002;25:572-584

25. Choi SH, Lee H, Park TG. PEGylation of G-CSF using cleavable oligolactic acid linkage. J Control Release. 2003;89:271-284.

26. Halpern W, Riccobene TA, Agostini H, et al. Albugranin, a recombinant human granulocyte colony stimulating factor (G-CSF) genetically fused to recombinant human albumin induces prolonged myelopoietic effects in mice and monkeys. Pharm Res. 2002;19:1720-1729.

27. Tanaka H, Satake-Ishikawa R, Ishikawa M, Matsuki S, Asano K Pharmacokinetics of recombinant human granulocyte colony-stimulating factor conjugated to polyethylene glycol in rats. Cancer Res. 1991;51: 3710-3714.

28. Choi SH, Park TG. G-CSF loaded biodegradable PLGA nanoparticles prepared by a single oil-in-water emulsion method. Int J Pharm. 2006; 311:223-228.

29. Maeda H, Nakagawa T, Adachi N, et al. Design of long-acting formulation of protein drugs with a double-layer structure and its application to rhG-CSF. J Control Release. 2003;91:281-297.

30. Gibaud S, Rousseau C, Weingarten C, et al. Polyalkylcyanoacrylate nanoparticles as carriers for granulocyte-colony stimulating factor (G-CSF). J Control Release. 1998;52:131-139.

31. Jin T, Zhu J, Wu F, Yuan W, Geng LL, Zhu H. Preparing polymer- based sustained-release systems without exposing proteins to water-oil or water-air interfaces and cross-linking reagents. $J$ Control Release. 2008;128:50-59.

32. Yuan W, Geng Y, Wu F, et al. Preparation of polysaccharide glassy nanoparticles with stabilization of proteins. Int J Pharm. 2009;366: $154-159$
33. Yuan W, Wu F, Geng Y, Xu S, Jin T. Preparation of dextran glassy particles through freezing-induced phase separation. Int J Pharm. 2007; 339:76-83.

34. Yuan W, Wu F, Guo M, Jin T. Development of protein delivery microsphere system by a novel s/o/o/w multi-emulsion. Eur J Pharm Sci. 2009;36:212-218.

35. Ren T, Yuan W, Zhao H, Jin T. Sustained-release polylactideco-glycolide microspheres loaded with pre-formulated protein polysaccharide nanoparticles. Micro Nano Lett. 2011;6:70-74.

36. Geng Y, Yuan W, Wu F, Chen J, He M, Jin T. Formulating erythropoietin-loaded sustained-release PLGA microspheres without protein aggregation. J Control Release. 2008;130:259-265.

37. Shirafuji N, Asano S, Matsuda S, Watari K, Takaku F, Nagata S. A new bioassay for human granulocyte colony stimulating factor (G-CSF) using murine myeloblastic NFS-60 cells as targets and estimation of its level in sera from normal healthy persons and patients with infections and hematological disorders. Exp Hematol. 1989;17:116-119.

38. Böyum A. Isolation of mononuclear cells and granulocytes from human blood. Scand J Clin Lab Invest Suppl. 1968;21:77-89.

39. Xu L, Wu F, Yuan W, Jin T. Controlled-release implant system formulated using biodegradable hemostatic gauze as scaffold. Int J Pharm. 2008;355:249-258.

40. Cleland JL, Jones, JS. Stable formulations of recombinant human growth hormone and interferon-gamma for microencapsulation in biodegradable microspheres. Pharm Res. 1996;13:1464-1475.

41. Schwendeman SP. Recent advances in the stabilization of protein encapsulated in injectable PLGA delivery systems. Crit Rev Ther Drug Carrier Syst. 2002;19:73-98.

42. Yuan W, Zhang Y, Wu F, et al. Preparation of protein-loaded sustainedrelease microspheres via 'solid-in-oil-in-hydrophilic oil-in-ethanol (S/O/hO/E)' emulsification. Colloids Surf B Biointerfaces. 2010;79: 326-333.

43. Ricci MS, Sarkar CA, Fallon EM, Lauffenburger DA, Brems DN. $\mathrm{pH}$ dependence of structural stability of interleukin-2 and granulocyte colony-stimulating factor. Protein Sci. 2003;12:1030-1038.

44. Wischke C, Schwendeman SP. Principles of encapsulating hydrophobic drugs in PLA/PLGA microparticles. Int J Pharm. 2008;364: 298-327.

45. Kim TH, Park TG. Critical effect of freezing/freeze-drying on sustained release of FITC-dextran encapsulated within PLGA microspheres. Int J Pharm. 2004;271:207-214.

46. Hu Z, Liu Y, Yuan W, Wu F, Su J, Jin T. Effect of bases with different solubility on the release behavior of risperidone loaded PLGA microspheres. Colloids Surf B Biointerfaces. 2011;86:206-211.
International Journal of Nanomedicine

\section{Publish your work in this journal}

The International Journal of Nanomedicine is an international, peerreviewed journal focusing on the application of nanotechnology in diagnostics, therapeutics, and drug delivery systems throughout the biomedical field. This journal is indexed on PubMed Central,

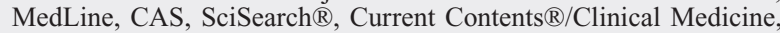

\section{Dovepress}

Journal Citation Reports/Science Edition, EMBase, Scopus and the Elsevier Bibliographic databases. The manuscript management system is completely online and includes a very quick and fair peer-review system, which is all easy to use. Visit http://www.dovepress.com/ testimonials.php to read real quotes from published authors. 